\title{
A Gestão do Conhecimento holística: análise de aderência do modelo de Mutuwa e Maiga
}

The holistic Knowledge Management: adherence analysis of the Mutuwa and Maita model

\section{La Gestión del Conocimiento holística: análisis de adherencia del modelo de Mutuwa y Maiga}

Fábio Corrêa $a^{1, a}$

fabiocontact@gmail.com | http://orcid.org/0000-0002-2346-0187

Fabrício Ziviani ${ }^{1, b}$

contato@fabricioziviani.com.br | http://orcid.org/oooo-0002-2705-846X

Dárlinton Barbosa Feres Carvalho ${ }^{2, c}$

darlinton@gmail.com | http://orcid.org/0000-0003-3844-0178

\footnotetext{
${ }^{1}$ Fundação Mineira de Educação e Cultura. Belo Horizonte, MG, Brasil.

2 Universidade Federal de São João del-Rei. São João del-Rei, MG, Brasil.

a Doutorado em Sistemas de Informação e Gestão do Conhecimento pela Fundação Mineira de Educação e Cultura.

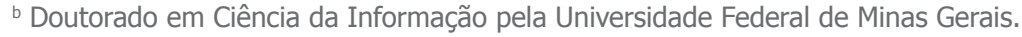

c Doutorado em Informática pela Pontifícia Universidade Católica - Rio de Janeiro.
}

\section{Resumo}

Há sinalizações acadêmicas para o desenvolvimento de modelos de gerenciamento do conhecimento pautados no paradigma holístico. Mutuwa e Maiga afirmam que não há um modelo unificador para o setor de saúde, o que permite inferir que o modelo desses autores se propõe ao atingimento desse feito. Assim, esta pesquisa se justifica pelo objetivo de analisar a aderência do modelo de Mutuwa e Maiga à Gestão do Conhecimento holística, de modo a identificar se esse modelo supre a lacuna de uma estrutura unificadora para este setor. Essa análise é realizada por meio de procedimentos metodológicos mistos, contemplando a abordagem qualitativa-quantitativa e a Análise de Conteúdo. Por resultado, há uma adesão de somente 15,4\% do modelo de Mutuwa e Maiga à Gestão do Conhecimento holística, conforme caracterização apresentada nessa pesquisa, permitindo inferir que a Gestão do Conhecimento não tem demonstrado direcionamento para uma abordagem orientada ao paradigma holístico. Assim, esta pesquisa contribui por fomentar o desenvolvimento de novos modelos de gerenciamento do conhecimento alicerçados no paradigma holístico, considerando os delineamentos aqui expostos.

Palavras-chave: Gestão do conhecimento; Paradigma holístico; Gestão do conhecimento holística; Mutuwa e Maiga; Análise de conteúdo. 


\begin{abstract}
There are academic signals for the development of knowledge management models based on the holistic paradigm. Mutuwa and Maiga affirm that there is no unifying model for the health sector, which allows to infer that the model of these authors is proposed to reach this achievement. Thus, this research is justified by the objective of analyzing the adherence of the model of Mutuwa and Maiga to Holistic Knowledge Management, in order to identify if this model bridged the gap of a unifying structure for this sector. This analysis is performed by means of mixed methodological procedures, including qualitative-quantitative approach and Content Analysis. As a result, the model of Mutuwa and Maiga adheres in $15.4 \%$ to the Holistic Knowledge Management, according to the characterization presented in this research, allowing to infer that Knowledge Management has not presented a direction towards a holistic paradigm-oriented approach. Thus, this research contributes to foster the development of new models of knowledge management based on the holistic paradigm, considering the designs presented here.
\end{abstract}

Keywords: Knowledge management; Holistic paradigm; Holistic knowledge management; Mutuwa and Maiga; Content analysis.

\title{
Resumen
}

Hay señales académicas para el desarrollo de modelos de gestión del conocimiento pautados en el paradigma holístico. Mutuwa y Maiga afirman que no hay un modelo unificador para el sector de salud, lo que permite inferir que el modelo de esos autores se propone al logro de ese hecho. Así, esta investigación se justifica por el objetivo de analizar la adherencia del modelo de Mutuwa y Maiga a la Gestión del Conocimiento holístico, para identificar si ese modelo suplía la laguna de una estructura unificadora para este sector. Este análisis se realiza a través de procedimientos metodológicos mixtos, contemplando el abordaje cualitativocuantitativo y el Análisis de Contenido. Por lo tanto, el modelo de Mutuwa y Maiga se adhiere en un 15,4\% puntos porcentuales a la Gestión del Conocimiento holístico, conforme caracterización presentada en esa investigación, permitiendo inferir que la Gestión del Conocimiento no ha presentado direccionamiento para un enfoque orientado al paradigma holística. Así, esta investigación contribuye por fomentar el desarrollo de nuevos modelos de gestión del conocimiento fundamentados en el paradigma holístico, considerando los delineamientos aquí expuestos.

Palabras chave: Gestión del conocimiento; Paradigma holístico; Gestión del conocimiento holístico; Mutuwa y Maiga; Análisis de contenido.

Contribuição dos autores:

Concepção, análise e redação do manuscrito: Fábio Corrêa.

Delineamento metodológico: Fabrício Ziviani.

Revisão crítica do conteúdo intelectual: Dárlinton Barbosa Feres Carvalho.

Declaração de conflito de interesses: não há.

Fontes de financiamento: Fundação Mineira de Amparo à Pesquisa do Estado de Minas Gerais (FAPEMIG) e Universidade FUMEC.

Considerações éticas: não há.

Agradecimentos/Contribuições adicionais: não há.

Histórico do artigo: submetido: 30 out. 2018 | aceito: 16 maio 2019 | publicado: 12 set. 2019.

Apresentação anterior: não houve.

Licença CC BY-NC atribuição não comercial. Com essa licença é permitido acessar, baixar (download), copiar, imprimir, compartilhar, reutilizar e distribuir os artigos, desde que para uso não comercial e com a citação da fonte, conferindo os devidos créditos de autoria e menção à Reciis. Nesses casos, nenhuma permissão é necessária por parte dos autores ou dos editores. 


\section{Introdução}

A Gestão do Conhecimento (GC) é uma temática pesquisada em diversos contextos, como saúde $^{1}$, Instituições de Ensino Superior ${ }^{2}$ e tecnologia ${ }^{3}$. Nesse âmbito, emergem os modelos de GC, sendo estruturas utilizadas para demonstrar os relacionamentos complexos entre as dimensões do gerenciamento do conhecimento ${ }^{4}$.

Liebowitz e Beckman ${ }^{5}$, Terra $^{6}$, Mehta ${ }^{7}$ e Batista ${ }^{8}$ são alguns modelos de GC que exprimem a relevância dessas estruturas para o contexto acadêmico e empresarial. Pela vertente empresarial, o estudo de Oliveira et al. ${ }^{9}$, que analisou os modelos de Liebowitz e Beckman ${ }^{5}$ e Mehta ${ }^{7}$, entre outros, fundamentou o desenvolvimento de um software de suporte à GC para uma empresa de base tecnológica, sendo este proposto $^{10} \mathrm{e}$ avaliado ${ }^{11}$ na prática organizacional.

Pela vertente acadêmica, os modelos de Terra ${ }^{6}$ e Batista ${ }^{8}$ são propostas brasileiras que fomentam novas pesquisas. Terra ${ }^{6}$ descreve sete dimensões do gerenciamento do conhecimento e alicerça a pesquisa de Miranda e Pilatti ${ }^{12}$, que promoveram a análise das dimensões deste modelo na indústria. Echternacht e Quandt ${ }^{13}$ se fundamentaram no modelo de Batista ${ }^{8}$ para avaliar o comportamento das secretarias municipais das capitais do Sul e Sudeste brasileiro e os responsáveis das câmaras municipais do Porto, Braga e Guimarães.

Em atenção à importância dos modelos de gerenciamento do conhecimento, Holsapple e Joshi ${ }^{14}$, Rubenstein-Montano et a ${ }^{15}$, Heisig ${ }^{16}$ e Fteimi ${ }^{17}$ analisaram o total de 270 modelos de GC e assinalaram que os modelos de GC devem ser conduzidos pela perspectiva do paradigma holístico. Esse paradigma demarca que as conexões entre as partes constituem o todo ${ }^{18-21} \mathrm{e}$, portanto, essas partes devem ser analisadas junto ao todo para que suas conexões sejam mantidas ${ }^{22}$.

No âmbito da GC, isso revela que as dimensões (partes) do gerenciamento do conhecimento devem ser consideradas em conjunto ${ }^{17}$ nos modelos de gerenciamento do conhecimento, pois assim as conexões entre elas serão mantidas, possibilitando atingir o holos, o íntegro, a totalidade do conhecimento e de sua gestão no contexto em que se aplica. Assim, um modelo de GC holístico deve apreciar todas as dimensões da GC para que se possa abordar essa forma de gestão por uma perspectiva abrangente.

Entretanto, há de se pesquisar se os modelos de GC da área de saúde têm atentado na necessidade de contemplar a GC mediante o paradigma holístico. Por essa problemática se estabelece o objetivo desta pesquisa: analisar a aderência do modelo de Mutuwa e Maiga ${ }^{23}$ à GC holística.

Wong e Aspinwall ${ }^{24} \mathrm{e}$ Rojas, Bermudez e Morales $^{1}$ abordaram a GC no contexto de saúde, sendo que esse segundo propôs um modelo de gerenciamento do conhecimento. Entretanto, Mutuwa e Maiga ${ }^{23}$ afirmam que não há um modelo unificador para esse setor, o que permite inferir que os autores se proponham a esse feito. Assim, o modelo instiga o intento de investigar o quão abrangente a proposta se apresenta perante a GC holística, justificando a escolha de sua análise por esta pesquisa.

A originalidade desta pesquisa se pauta por buscar compreender, no âmbito da saúde, como a GC tem avançado rumo a uma abordagem holística, mister para o desenvolvimento de modelos de gerenciamento do conhecimento, conforme assinalado por estudos internacionais e nacionais ${ }^{14-17,22}$. No âmbito local, este estudo apresenta relevância por evidenciar as dimensões críticas para o sucesso da GC holística em saúde, municiando outros pesquisadores a contemplarem estas dimensões para um gerenciamento do conhecimento íntegro neste setor.

Para atingir esse objetivo, primeiramente são consolidadas as dimensões (partes) que constituem a GC holística (subseção 1.1). Em sequência, os métodos a serem utilizados para analisar o modelo Mutuwa e Maiga $^{23}$ são elucidados (seção 2). Por conseguinte, o modelo em questão é analisado e seu grau de aderência à GC holística é quantificado (seção 3). Em continuidade, as considerações finais acerca desta pesquisa são apresentadas (seção 4), e as referências utilizadas findam este estudo. 


\section{Dimensões da gestão do conhecimento holística}

Dimensões são partes que constituem e dão forma a algo. As empresas são constituídas por pessoas, sendo uma dimensão que conforma o ambiente empresarial. Não obstante, a área de recursos humanos apresentase como outra dimensão organizacional, pois essa tem em seu cerne as pessoas e o desafio de geri-las em prol do conhecimento tácito9. Ambas compõem e dão forma ao contexto empresarial em suas diferentes extensões.

Na perspectiva dos fatores críticos de sucesso, as dimensões da GC são áreas que garantem o desempenho bem-sucedido de uma iniciativa ${ }^{25-30}$. Assim, para uma GC holística, tais dimensões devem ser contempladas em conjunto num modelo de gerenciamento do conhecimento. Desse modo, as dimensões que conformam o gerenciamento do conhecimento orientado no paradigma holístico foram consolidadas por meio de uma revisão sistemática da literatura.

A pesquisa foi promovida em 12 de agosto de 2017 na base Scopus, valendo-se dos termos 'knowledge management' and 'critical success factors' no título e sem recorte temporal. Foram obtidas 48 publicações, que foram analisadas de modo a manter somente as que fundamentam teoricamente as dimensões críticas para $o$ sucesso da GC. Por resultado, foram consideradas 18 publicações científicas ${ }^{24-41}$ com temporalidade de 1997 a 2015. Após a interpretação teórica das dimensões da GC holística, foram consolidadas 13 dimensões, a saber:

Estratégia: alinhar a estratégia de GC à organizacional e comunicá-la à organização para que os funcionários compreendam o intento da $\mathrm{GC}^{31,34,36,40}$;

Liderança e suporte da alta administração: apoiar a GC no direcionamento de conhecimentos relevantes, nos aspectos financeiros e materiais, concessão de tempo para desenvolver as atividades de conhecimento, bem como homogeneizar a mensagem da GC $25,29-28,32,36$;

Equipe de gestão do conhecimento: definir responsabilidades e perfis para o estabelecimento de uma equipe de GC na organização $0^{25,28,32,35,38}$;

Recursos (financeiro, humano, material e tempo): avaliar e conceder recursos materiais, humanos, financeiros e de tempo para a operacionalização da $\mathrm{GC}^{25,30,36,40}$;

Processos e atividades: são o centro da GC por elucidarem o manuseio e o que pode ser feito com o conhecimento, sendo o núcleo da criação de uma empresa voltada ao conhecimento ${ }^{25,33,40}$;

Gestão de recursos humanos: contratar e capacitar as pessoas perante as lacunas de conhecimento, bem como desenvolver políticas considerando a retenção dos indivíduos ${ }^{30,33}$;

Treinamento e educação: treinar as pessoas para homogeneizar vocabulário, conceitos e objetivo da GC; estabelecer o propósito e a equipe de GC; prover habilidades para as atividades de conhecimento e uso das ferramentas de GC; aceitar erros para aprendizado por sucesso e falha; e educar os funcionários para os quesitos anteriores ${ }^{28,30-32,36,40}$;

Motivação: desenvolver planos de avaliação e de carreira, sistema de motivação (recompensas, reconhecimento e gratificações) e sinalizar à gestão de recursos humanos práticas motivacionais que fomentem a criação e compartilhamento do conhecimento ${ }^{25,28-29,38}$;

Trabalho em equipe: fomentar o trabalho em equipe para promover o compartilhamento e criação de novos conhecimentos ${ }^{25,34}$;

Cultura: estabelecer uma cultura colaborativa e benéfica à GC por meio da tolerância a erros, de forma a reconhecer o potencial de aprendizado por meio deles $33,35-36,38$;

Tecnologia da Informação: a tecnologia é um facilitador eficaz de manipulação (captura, armazenamento e divulgação) do conhecimento e para conectar as pessoas, sendo indiscutível sua relevância para a GC. No entanto é imperativo frisar que essa deve ser percebida como um meio para os processos da GC33,36,38,40;

Mensuração: definir indicadores e métricas para mensurar os objetivos da GC, diagnósticos organizacionais e comunicar à alta administração os resultados aferidos ${ }^{25,28,32-33,36}$; 
Projeto Piloto: planejar projetos piloto para investidas em menor âmbito organizacional, permitindo identificar melhores práticas e elevar o potencial de acerto em investidas de maior âmbito ${ }^{31,34}$.

Deste modo, as 13 dimensões acima fundamentadas constituem o que é crítico para o sucesso do gerenciamento do conhecimento. Perante o paradigma holístico, estas devem ser contempladas conjuntamente em um modelo de $\mathrm{GC}^{17}$, de modo a manter as conexões adjacentes entre elas. Estas dimensões constituem as unidades de registro a serem utilizadas na análise do modelo de Mutuwa e Maiga ${ }^{23}$.

\section{Método}

Esta pesquisa se caracteriza pela abordagem qualitativa-quantitativa. A abordagem qualitativa é empregada pelo uso da Análise de Conteúdo ${ }^{42}$, que consiste em "Um conjunto de técnicas de análise das comunicações visando obter, por procedimentos, sistemáticos e objectivos de descrição do conteúdo das mensagens, indicadores (quantitativos ou não) que permitam a inferência de conhecimentos relativos às condições de produção/ recepção (variáveis inferidas) destas mensagens"42.

Por outros termos, busca-se interpretar o conteúdo escrito do modelo de Mutuwa e Maiga ${ }^{23}$ e analisálo de modo a promover inferências quanto à sua adesão à GC holística. Para produzir tais inferências, é necessário estabelecer, durante a análise, as unidades de registro, que são os elementos a serem identificados no texto para posterior ordenação, categorização e contagem frequêncial ${ }^{42}$.

As unidades de registro constituem as 13 dimensões da GC holística, consolidadas e fundamentadas na seção anterior desta pesquisa. Desse modo, busca-se inferências da existência ou ausência destas dimensões no modelo analisado. Visando a um maior rigor metodológico na aplicação da Análise de Conteúdo², as inferências apresentadas serão fundamentadas e expostas mediante a interpretação íntegra do modelo, de modo a permitir a compreensão delas e a manutenibilidade dos resultados apreendidos.

Após analisar qualitativamente o modelo de Mutuwa e Maiga ${ }^{23}$, de forma a identificar a presença ou ausência das unidades de registro acima mencionadas, essas serão contabilizadas pela abordagem quantitativa a fim de revelar o grau percentil de adesão do referido modelo à GC holística.

\section{Resultados}

Nesta seção é promovida a Análise de Conteúdo qualitativa do modelo de Mutuwa e Maiga ${ }^{23}$ para que, em seguida, seja quantificada sua aderência à GC holística.

A área de saúde se apresenta como um campo baseado no conhecimento que conecta vários setores, entre eles, hospitais, clínicas e farmácias. A tomada de decisões nesse setor é aprimorada quando o conhecimento certo pode ser obtido no momento certo e, especificamente no campo pediátrico, que visa à saúde infantil para atingimento do potencial do indivíduo quando adulto, o conhecimento se torna vital ${ }^{23}$.

Na pediatria, assim como em outros campos da saúde, a exemplo do turismo de saúde ${ }^{1}$, há vários domínios da medicina como radiologia e cirurgia que, consequentemente, envolvem organizações governamentais e não governamentais, instituições hospitalares e financeiras, escolas e comunidades locais como atores desse contexto ${ }^{23}$. Assim, para Mutuwa e Maiga, "o sucesso dos cuidados de saúde depende criticamente da coleta, análise e troca contínua de conhecimento clínico e médico dentro e entre fronteiras organizacionais”²3.

Nesse cenário, Mutuwa e Maiga ${ }^{23}$ inserem a GC como uma abordagem que tende a melhorar os resultados referentes à saúde pediátrica, mas, segundo os pesquisadores, a GC nesse campo é fragmentada e não apresenta um modelo unificador. Devido à importância da pediatria para a vida adulta e pela ausência de uma abordagem concisa de GC nesse campo, os pesquisadores se propõem a "criar um modelo de Gestão do Conhecimento que apoie a integração da geração intensiva de conhecimento à prática pediátrica de cuidados de saúde aplicada" ${ }^{23}$ no país africano de Uganda. 
Para a conformação do modelo, os pesquisadores fizeram uso de entrevistas e questionário aplicados a pediatras atuantes em instituições hospitalares e de saúde. Em sequência foram realizadas análises estatísticas sobre os dados, visando capturar os elementos inerentes à GC e sua significância. Por resultado, Mutuwa e Maiga ${ }^{23}$ ajustam as pessoas, tecnologias e a GC, com suas fases cíclicas de criação, aquisição, armazenamento e compartilhamento, como elementos que influenciam e integram o conhecimento pediátrico que, por sua vez, tende a promover a melhoria de entrega de cuidados pediátricos (Figura 1).

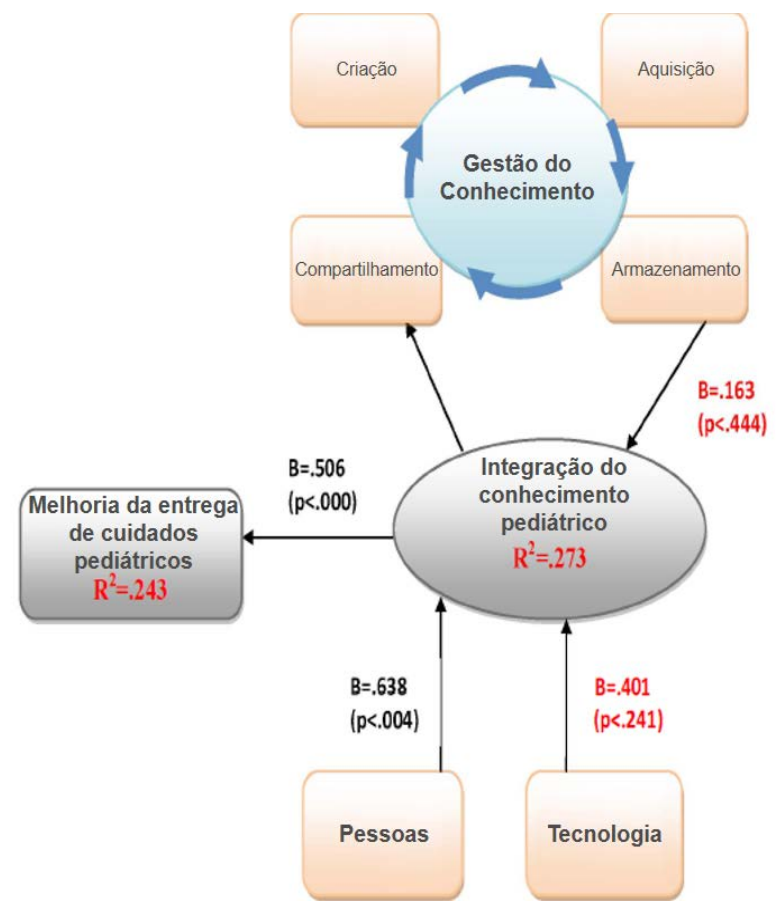

Figura 1 - Um modelo de gestão do conhecimento para a prática de cuidados de saúde pediátrica Fonte: Adaptado de Mutuwa e Maiga23

As fases cíclicas no modelo se associam à dimensão Processos e atividades da GC holística. Os processos e atividades são o centro da GC por serem manuseadores do conhecimento e estão relacionados ao ciclo de vida desse ativo. Tais processos elucidam o que pode ser feito com o conhecimento ${ }^{33,40}$ e são o núcleo da criação de uma empresa voltada ao conhecimento ${ }^{25}$.

A dimensão tecnologia do modelo de Mutuwa $\mathrm{e} \mathrm{Maiga}^{23}$ se alinha à dimensão Tecnologia da Informação da GC holística. A relevância da tecnologia para a GC é indiscutível ${ }^{33}$; no entanto, é imperativo frisar que essa deve ser percebida como um meio para os processos da GC e não como uma solução definitiva ${ }^{38,40}$. Mutuwa e Maiga afirmam que "[...] melhorar a assistência pediátrica a integração do conhecimento pediátrico deve ser levada em consideração com todos os seus colaboradores (armazenamento de conhecimento e tecnologia, com foco principal em pessoas)" ${ }^{23}$.

Essa afirmativa é alicerçada no fato de que a tecnologia e o armazenamento não apresentam influência significativa na integração do conhecimento pediátrico, conforme dados estatísticos do modelo. A tecnologia e o armazenamento estão um para o outro, pois a tecnologia é um facilitador eficaz de manipulação (captura, armazenamento e divulgação) do conhecimento ${ }^{36}$.

A percepção dos entrevistados por Mutuwa e Maiga $^{23}$ quanto a esses itens corrobora com o estudo realizado por Wong e Aspinwall ${ }^{24}$. Também ambientados no setor de saúde, Wong e Aspinwall ${ }^{24}$ aferiram que os médicos não percebem a TI como fator relevante para a GC, uma vez que assumem formas convencionais de compartilhamento do conhecimento médico. A percepção dos entrevistados nos estudos de Wong e Aspinwall $^{24}$ e Mutuwa e Maiga ${ }^{23}$ demarca e enfatiza o papel da tecnologia como mediador na GC. 
Mutuwa e Maiga pontuam a relevância de promover a "análise e troca contínua de conhecimento clínico e médico dentro e entre fronteiras organizacionais”23; entretanto, essas fronteiras não são expressas no modelo. Os pesquisadores também assinalam a ausência de um modelo unificador no campo pediátrico, mas o framework proposto não parece suprir essa lacuna e sim lançar uma luz para futuras pesquisas nesse contexto.

Mutuwa e Maiga $^{23}$ usam a quantificação para criar o modelo e avaliar sua adesão, ambos no contexto pediátrico de saúde. O estudo se limita a posicionar as dimensões da GC em um arquétipo, não apresentando a descrição das dimensões ou como operacionalizá-las na prática. Por síntese, esse modelo apresenta as seguintes dimensões da GC holística:

a) Estratégia: não especificado;

b) Liderança e suporte da alta administração: não especificado;

c) Equipe de gestão do conhecimento: não especificado;

d) Recursos (financeiro, humano, material e tempo): não especificado;

e) Processos e atividades: sim. Criação, aquisição, armazenamento e compartilhamento;

f) Gestão de recursos humanos: não especificado;

g) Treinamento e educação: não especificado;

h) Motivação: não especificado;

i) Trabalho em equipe: não especificado;

j) Cultura: não especificado;

k) Tecnologia da Informação: sim;

l) Mensuração: não especificado;

Mediante a análise promovida sobre o modelo de Mutuwa e Maiga ${ }^{23}$, as dimensões, acima ponderadas, são dispostas na Figura 2, que exprime a adesão do referido modelo às dimensões da GC holística concernentes a essa pesquisa. A presença de uma dimensão a posiciona na escala 1 (extremidade externa) do gráfico e sua ausência a assinala na escala o (centro).

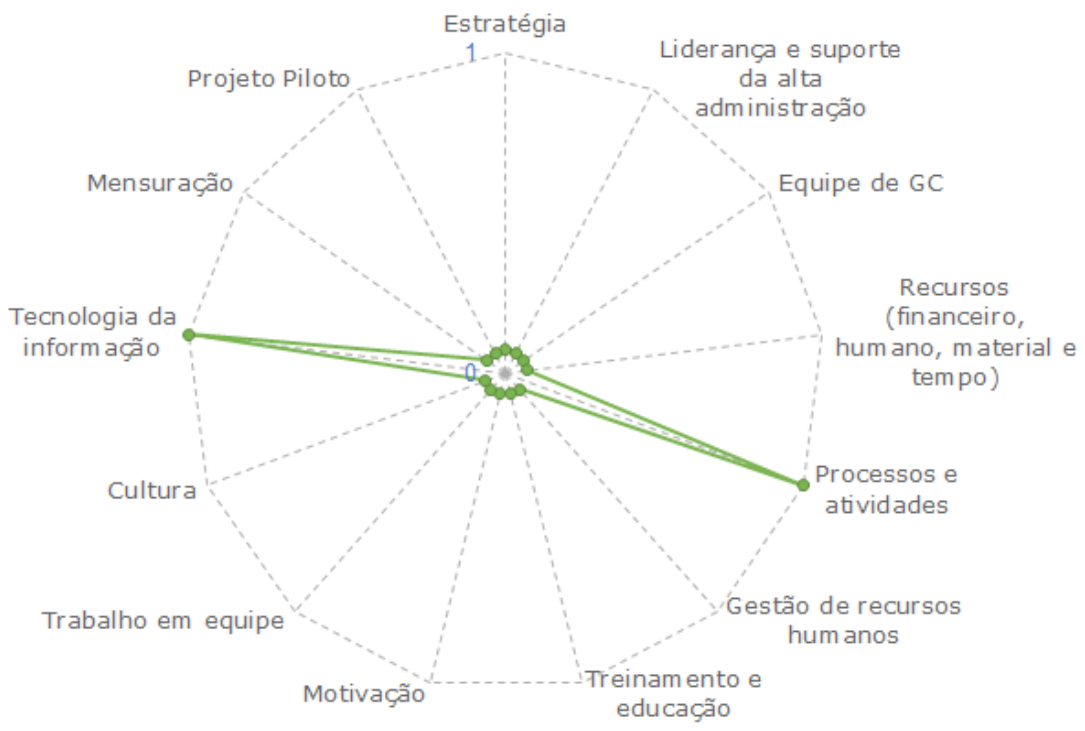

Figura 2 - Adesão do modelo de Mutuwa e Maiga ${ }^{23}$ às dimensões da gestão do conhecimento holística. Fonte: Os autores (2019).

Assim, o modelo de Mutuwa e Maiga ${ }^{23}$ contempla as dimensões Processos e atividades e Tecnologia da informação, remetendo-o ao percentual de 15,4\% (2 do total de 13 dimensões) de adesão ao que considera por GC holística, conforme caracterização apresentada nesta pesquisa. 


\section{Considerações finais}

Esta pesquisa teve por objetivo analisar a aderência do modelo de Mutuwa e Maiga ${ }^{23}$ à GC holística. A GC holística foi delineada e suas dimensões foram apresentadas de forma a constituir um alicerce para a análise do modelo. Mediante procedimentos metodológicos mistos, o referido modelo foi analisado e seu grau de adesão à GC holística foi expresso em percentis.

Embora Mutuwa e Maiga ${ }^{23}$ anunciem que a GC no setor de saúde seja fragmentada e não disponha de um modelo unificador, os pesquisadores não apresentam um modelo que seja abrangente de forma a sanar a lacuna apresentada. Em verdade, os autores apresentam dimensões de senso comum, já apontadas em outros estudos, como na pesquisa de Wong e Aspinwall ${ }^{24}$ e no modelo de Rojas, Bermudez e Morales ${ }^{1}$.

É importante salientar que esta pesquisa não apresenta o intento de atribuir mérito ou demérito ao modelo analisado. O objeto é examinar o quanto este modelo se orienta ao gerenciamento do conhecimento holístico, e como tal, outras interpretações não devem ser realizadas, mediante a leitura desta pesquisa, sob a ótica de enfraquecer a proposta dos autores.

Por resultado, somente por 15,4 pontos percentuais há uma adesão do modelo de Mutuwa e Maiga ${ }^{23}$ à GC holística, conforme caracterização apresentada nesta pesquisa, permitindo inferir que a lacuna de um modelo de GC, amparado no paradigma holístico, ainda não foi suprida.

Entretanto, esse resultado não é conclusivo, pois somente um modelo foi analisado, sendo essa a limitação desta pesquisa. Dessa forma, uma sugestão de pesquisa futura é promover a análise de outros modelos de GC de modo a permitir identificar se há propostas sendo conduzidas perante o paradigma holístico no campo de saúde. Isso tende a revelar o quanto o gerenciamento do conhecimento tem caminhado para uma abordagem mais totalitária nesse setor, de forma a influenciar pesquisadores para que desenvolvam modelos que considerem os delineamentos expostos nesta pesquisa.

\section{Referências}

1. Rojas LAR, Bermudez GMT, Morales AAO. A knowledge management model applied to health tourism in Colombia [Internet]. In: Anais da $7^{a}$ International Conference on Knowledge Management in Organizations: Service and Cloud Computing; 2002; Heidelber, Berlin, 2013 [cited 2019 jul. 22]. p. 537546. doi: http://dx.doi.org/10.1007/978-3-642-30867-3 48.

2. Farías EB, Mercado MTC, González MJG. Modelo de gestión de conocimiento para el desarrollo de posgrado: estudio de caso. Revista Electrónica de Investigación Educativa [Internet]. 2016 [citado em 2018 nov. 19]; 18(1):128-139. Disponível em: http://www.scielo.org.mx/pdf/redie/v18n1/v18n1a9.pdf.

3. Moscoso-Zea $\mathrm{O}$ et al. Knowledge management framework using enterprise architecture and business intelligence. In: Anais da ICEIS 2016: $18^{\mathrm{a}}$ International Conference on Enterprise Information Systems; 2016; Rome, Italy, 2016. p. 244-249.

4. Cajueiro JLG. Modelo de gestão do conhecimento para instituições de ensino superior. [tese]. Recife: Universidade Federal de Pernambuco; 2008. [citado em 2018 nov. 19]. Disponível em: https:// repositorio.ufpe.br/handle/123456789/4985.

5. Liebowitz J, Beckman TJ. Knowledge organizations: what every manager should know. CRC Press; 1998.

6. Terra JCC. Gestão do conhecimento: o grande desafio empresarial. 5 ed. Rio de Janeiro: Elsevier; 2005.

7. Mehta N. Successful knowledge management implementation in global software companies. Journal of Knowledge Management [Internet]. 2008 [cited 2018 nov. 19]; 12(2):42-56. doi: https://doi. org/10.1108/13673270810859505.

8. Batista FF. Modelo de gestão do conhecimento para a administração pública brasileira: como implementar a gestão do conhecimento para produzir resultados em benefício do cidadão. Brasília: Instituto de Pesquisa Econômica Aplicada; 2012. 
9. Oliveira $\mathrm{M}$ et al. Espiral do conhecimento em frameworks de gestão do conhecimento: o caso de duas organizações em Portugal. Perspectivas em Ciência da Informação [Internet]. 2010 [citado em 2018 nov. 19]; 15(3):155-175. Disponível em: http://portaldeperiodicos.eci.ufmg.br/index.php/pci/article/ view/877/781.

10. Antunes $\mathrm{J}$ et al. Criação de sistema para apoio a gestão do conhecimento em empresa de desenvolvimento de software. In: Anais do $18^{\circ}$ SBSI: Simpósio Brasileiro de Sistemas de Informação; 2016; Brasil, Lavras, 2016. p. 496-503.

11. Antunes $\mathrm{J}$ et al. Avaliação de um sistema para gestão do conhecimento de uma empresa de desenvolvimento de software. In: Anais do $18^{\circ}$ SBSI: Simpósio Brasileiro de Sistemas de Informação; 2016; Brasil, Lavras, 2016. p. 512-519.

12. Miranda ITP, Pilatti LA. Análise das sete dimensões da gestão do conhecimento em uma indústria do setor metal-mecânico. Revista Tecnologia e Sociedade [Internet]. 2014 [citado em 2018 nov. 19]; 10(20):121-135. doi: http://dx.doi.org/10.3895/rts.v10n20.2646.

13. Echternacht THS, Quandt, CO. A gestão do conhecimento como suporte ao processo estratégico na administração pública municipal: um estudo comparativo de casos no Brasil e em Portugal. Perspectivas em Gestão \& Conhecimento [Internet]. 2017 [citado em 2018 nov. 19]; 7(1):35-49. Disponível em: http://www.periodicos.ufpb.br/index.php/pgc/article/view/32973/17291.

14. Holsapple CW, Joshi KD. Description and analysis of existing knowledge management frameworks [Internet]. In: Systems Sciences, 1999. In: Anais do $32^{\circ}$ HICSS: Annual Hawaii International Conference on IEEE; 1999 [cited 2019 jul. 22]; p.15. doi: https://doi.org/10.1109/HICSS.1999.772796.

15. Rubenstein-Montano $B$ et al. A systems thinking framework for knowledge management. Decision Support Systems [Internet]. 2001 [cited 2018 nov. 19]; 31(1):5-16. doi: https://doi.org/10.1016/S01679236(00)00116-0.

16. Heisig P. Harmonisation of knowledge management - comparing $160 \mathrm{KM}$ frameworks around the globe. Journal of Knowledge Management [Internet]. 2009 [cited 2018 nov. 19]; 13(4):4-31. doi: https://doi. org/10.1108/13673270910971798.

17. Fteimi N. Analyzing the literature on knowledge management frameworks: Towards a normative knowledge management classification schema. In: Anais do $23^{\circ}$ ECIS: European Conference on Information Systems; 2015; Münster, Germany, 2015. p. 1-16.

18. Weil P. O novo paradigma holístico: ondas a procura do mar. In: Brandão D, Crema R, organizadores. $O$ novo paradigma holístico: ciência, filosofia, arte e mística. São Paulo: Summus; 1991. p. 14-38.

19. Capra F. A teia da vida: uma nova compreensão científica dos sistemas vivos. 9. ed. São Paulo: Cultrix; 2000.

20. Morin E. Ciência com consciência. 8. ed. Rio de Janeiro: Bertrand Brasil; 2005.

21. Crema R. Introdução à visão holística: breve relato de viagem do velho ao novo paradigma. 6. ed. São Paulo: Summus; 2015.

22. Pereira MF. A gestão organizacional em busca do comportamento holístico. In: Angeloni MT, organizador. Organizações do conhecimento: infra-estrutura, pessoas e tecnologia. São Paulo: Saraiva; 2017. p. 2-28.

23. Mutuwa PL, Maiga G. A knowledge management model for pediatric health care practice [Internet]. In: Anais do $2^{\circ}$ PACT: Pan African International Conference on Science, Computing and Telecommunications; 2014 [cited 2019 jul. 22]; Arusha, Tanzânia, 2014. p. 40-45. doi: http://dx.doi. org/10.1109/SCAT.2014.7055134.

24. Wong KY, Aspinwall E. An empirical study of the important factors for knowledge-management adoption in the SME Sector. Journal of Knowledge Management [Internet]. 2005 [citado em 2018 nov. 19]; 9(3):64-82. doi: https://doi.org/10.1108/13673270510602773.

25. Sedighi M, Zand F. Knowledge management: Review of the Critical Success Factors and development of a conceptual classification model [Internet]. In: Anais do International Conference on ICT and Knowledge Engineering; 2012 [cited 2019 jul. 22]; Bangkok, Thailand; 2012. p. 1-9. doi:http://dx.doi. org/10.1109/ICTKE.2012.6408553. 
26. Anggia P et al. Identifying critical success factors for knowledge management implementation in organization: a survey paper [Internet]. In: Anais do ICACSIS: International Conference on Advanced Computer Science and Information Systems; 2013 [cited 2019 jul. 22]; Bali, Indonesia; 2013. p. 83-88, 2013. doi: http://doi.org/10.1109/ICACSIS.2013.6761556.

27. Naghavi M, Dastaviz AH, Nezakati H. Relationships among critical success factors of knowledge management and organizational performance. Journal of Applied Sciences [Internet]. 2013 [cited 2018 nov. 19]; 13(5):755-759. doi: http://doi.org/10.3923/jas.2013.755.759.

28. Arif MJ, Shalhoub MHB. Critical success factors with its effective role in knowledge management initiatives in public and private organizations in saudi Arabia: experts perspectives. Life Science Journal [Internet]. 2014 [cited 2018 nov. 19]; 11(6):636-645. Disponível em: https://bit.ly/32JwBQg.

29. Zieba M, Zieba K. Knowledge management critical success factors and the innovativeness of KIBS companies. Engineering Economics [Internet]. 2014 [citado em 2018 nov. 19]; 25(4):458-465. doi: http://dx.doi.org/10.5755/j01.ee.25.4.6494.

30. Kumar S, Singh V, Haleem A. Critical success factors of knowledge management: modelling and comparison using various techniques. International Journal of Industrial and Systems Engineering [Internet]. 2015 [cited 2018 nov. 19]; 21(2):180-206. doi: http://doi.org/10.1504/IJISE.2015.071508.

31. Skyrme D, Amidon D. The knowledge agenda. Journal of Knowledge Management [Internet]. 1997 [cited 2018 nov. 19]; 1(1):27-37. doi: http://dx.doi.org/10.1108/13673279710800709.

32. Davenport TH, De Long DW, Beers MC. Successful knowledge management projects. Sloan Management Review [Internet]. 1998 [cited 2018 nov. 19]; 39(2):43-57. Disponível em: https://www.researchgate. net/profile/Thomas Davenport2/publication/200045855 Building Successful Knowledge Management Projects/links/53db93a40cf216e4210bf847.pdf.

33. Wong KY. Critical success factors for implementing knowledge management in small and medium enterprises. Industrial Management \& Data Systems [Internet]. 2005 [cited 2018 nov. 19]; 105(3):261279. doi: http://dx.doi.org/10.1108/02635570510590101.

34. Akhavan $\mathrm{P}$, Jafari $\mathrm{M}$, Fathian $\mathrm{M}$. Critical success factors of knowledge management systems: A multi-case analysis. European Business Review [Internet]. 2006 [cited 2018 nov. 19]; 18(2):97-113. doi: http://doi. org/10.1108/09555340610651820.

35. Lin YC, Lin LK. Critical success factors for knowledge management studies in construction. In: Anais do $23^{\circ}$ ISARC: International Symposium on Robotics and Automation in Construction; 2006; Tóquio, Japão; 2006. p. 768-772.

36. Al-Mabrouk K. Critical success factors affecting knowledge management adoption: A review of the literature [Internet]. In: Anais do IITFALSO: Innovations in Information Technology; 2006 [cited 2019 jul. 22]; Dubai, Arábia Saudita; 2006. doi: http://dx.doi.org/10.1109/INNOVATIONS.2006.301888.

37. Gai $\mathrm{S}, \mathrm{Xu} \mathrm{C}$. Research of critical success factors for implementing knowledge management in China [Internet]. In: Anais do $4^{\circ}$ ICIII: International Conference on Information Management, Innovation Management and Industrial Engineering; 2009 [cited 2019 jul. 22]; Xi'an, China; 2009. p.561-564. doi: http://doi.org/10.1109/ICIII.2009.594.

38. Valmohammadi C. Investigation and assessment of critical success factors of knowledge management implementation in Iranian small-to-medium sized enterprises. Journal of Applied Sciences [Internet]. 2010 [cited 2018 nov. 19]; 10(19):2290-2296. Disponível em: http://docsdrive.com/pdfs/ansinet/ jas/2010/2290-2296.pdf.

39. Altaher AM. Critical success factors of implementation knowledge management process. In: Anais do i-Society: International Conference on Information Society; 2010; Londres, Inglaterra; 2010. p. 340-348.

40. Abbaszadeh MA, Ebrahimi M, Fotouhi H. Developing a causal model of critical success factors for knowledge management implementation [Internet]. In: Anais do ICEMT 2010: International Conference on Education and Management Technology; 2010 [cited 2019 jul. 22]; Cairo, Egito; 2010. p.701-705. doi: http://dx.doi.org/10.1109/ICEMT.2010.5657563.

41. Wai YM, Hong ANH, Din, SB. Critical success factors and perceived benefits of knowledge management implementation: towards a conceptual framework. Australian Journal of Basic and Applied Sciences [Internet]. 2011 [cited 2018 nov. 19]; 5(10):754-760. Disponível em: http://ajbasweb.com/old/ ajbas/2011/October-2011/754-760.pdf. 